Bolyai Society - Springer-Verlag

\title{
HEREDITARILY EXTENDED PROPERTIES, QUASI-RANDOM GRAPHS AND NOT NECESSARILY INDUCED SUBGRAPHS
}

\author{
MIKLÓS SIMONOVITS and VERA T. SÓS*
}

Received March 7, 1997

Dedicated to the memory of Paul Erdös

Recently much attention has been focused on the theory of quasi-random graph and hypergraph properties. The class of quasi-random graphs is defined by certain equivalent graph properties possessed by random graphs. We shall investigate properties $\mathbf{P}$ which do not imply quasi-randomness for sequences $\left(G_{n}\right)$ of graphs on their own, but do imply if they hold not only for the whole graph $G_{n}$ but also for every sufficiently large subgraph of $G_{n}$. Here the properties are strongly connected to counting not necessarily induced subgraphs of a given type, while in a subsequent paper we shall investigate the properties connected with counting induced subgraphs.

\section{Notation}

Below we consider simple graphs, that is, loops and multiple edges will be excluded. Given a graph $G, V(G)$ and $E(G)$ will denote the vertex- and edge set of the graph $G, v(G)$ and $e(G)$ the number of vertices and edges, respectively. The (first) subscript in case of graphs will always denote the number of vertices. So $G_{n}, S_{n}$ are always graphs of order $n . K_{r}, C_{r}$ and $P_{r}$ denote the complete graph, the cycle and the path on $r$ vertices, respectively. Given a subset $X$ of $V(G), e(X)$ denotes the number of edges of the subgraph induced by $X$, and $G[X]$ denotes the subgraph of $G$ spanned by $X$. Given two disjoint subsets $X$ and $Y$ in $V(G)$, $e(X, Y)$ denotes the number of edges between $X$ and $Y$. To avoid complicated notations we sometimes allow ourselves to denote the same graph $L_{\nu}$ also by $L$ (mostly when it appears in subscripts or superscripts). Given a graph $G, N_{G}(x)$ (mostly abbreviated to $N(x)$ ) denotes the set of neighbors of $x$ in $G$.

Mostly we shall have a sample graph $L_{\nu}$ with $V\left(L_{\nu}\right)=\left\{x_{1}, x_{2}, \ldots, x_{\nu}\right\}$ and a graph $G_{n}$ with a copy of $L_{\nu}$ in it whose vertices are $\left\{y_{1}, y_{2}, \ldots, y_{\nu}\right\}$.

- A labelled induced copy of $L_{\nu}$ in $G_{n}$ means a function $\psi: V\left(L_{\nu}\right) \rightarrow V\left(G_{n}\right)$ mapping different $x_{i}$ 's into different $y_{t}$ 's, where $\left(\psi\left(x_{i}\right), \psi\left(x_{j}\right)\right) \in E\left(G_{n}\right)$ iff $\left(x_{i}, x_{j}\right) \in$ $E\left(L_{\nu}\right)$, while

Mathematics Subject Classification (1991): 05C80

* Research supported by OTKA N1909. 
- a not necessarily induced labelled copy (below abbreviated to "NNI") means a function $\psi$ where we assume only that if $\left(x_{i}, x_{j}\right) \in E\left(L_{\nu}\right)$, then $\left(\psi\left(x_{i}\right), \psi\left(x_{j}\right)\right) \in$ $E\left(G_{n}\right)$.

Now, denote the number of labelled

induced

copies of $L_{\nu} \subseteq G \quad$ by $\quad \mathrm{N}^{*}\left(L_{\nu} \subseteq G\right)$

and the number of

not necessarily induced copies of $L_{\nu} \subseteq G$ by $\mathbf{N}\left(L_{\nu} \subseteq G\right)$,

respectively.

To indicate that two quantities $A_{n}$ and $B_{n}$ are approximately equal, i.e. $A_{n} / B_{n} \rightarrow 1$ as $n \rightarrow \infty$, we shall use the notation $A_{n} \sim B_{n}$.

Remark. All the theorems of this paper are formulated for labelled graphs, however, all our results easily extend to unlabelled graphs.

\section{Introduction}

One of the important questions of modern mathematics and modern computer science is, how randomlike objects can be generated in nonrandom ways and when an individual event could be considered random, and in which sense. There are several reasons why this question has become extremely important (e.g., in case of sequences of numbers).

- In many of our algorithms we can speed up the calculations by using random numbers; however, whenever we try to generate "random numbers" by computers, they are not truely random, they only look randomlike.

- In the applications of the Monte Carlo method one needs to know if the random number generator used yields a sequence that can be regarded "random" or not.

- The question to decide if a sequence is randomlike or not is important also in mathematical statistics.

- The fundamental problems of probability theory and some practical applications also need this clarification.

Of course, there are many other reasons why studying random objects is an exciting and important question. Thus e.g., the study of uniformly distributed sequences is also strongly related to this topic. The literature on these questions is quite extensive.

At this point we could ask:

"What is the meaning of that a sequence looks randomlike?"

This question has several nonequivalent answers. It was answered in some sense by the Kolmogorov complexity theory [10] and in some completely different sense it was made fairly precise in the recent complexity theory of randomized algorithms. In this later case a sequence is considered to be randomlike if there 
is no fast algorithm (or, alternatively, no small computing circuit,...) which can distinguish this sequence from a true random sequence.

A. Thomason, [17], [18] and F. R. K. Chung, R. L. Graham, and R. M. Wilson, [2], gave some characterization of randomlike graph sequences, Chung and Graham [3] extended this to hypergraph sequences, P. Frankl, V. Rödl and R. Wilson [9] gave some characterizations of "randomlike" matrix sequences.

In [2] a class of graph properties were considered, all possessed by random graphs and at the same time equivalent to each other in some well-defined sense. Properties like these are called quasi-random properties. Quite a few papers were launched to clarify the basic quasi-random properties for graphs, and also for hypergraphs, matrices, or for subsets of integers, [2], [3], [5], [6], [7], [9]. There are some interesting negative examples of properties which one would think to be quasi-random, while they are not.

In this paper we shall investigate those properties $\mathbf{P}$ which do not imply quasi-randomness of sequences $\left(G_{n}\right)$ of graphs on their own, but do imply if they are assumed not only for the whole graphs $G_{n}$ but also for every sufficiently large induced subgraph $F_{h} \subseteq G_{n}$. Such properties will be called Hereditarily Extended Properties, or shortly, Hereditary Properties. To consider such extensions is motivated by the fact that sufficiently large subgraphs of randomlike graphs must also be randomlike. In other words, randomness is a "hereditary property".

Let $\mathscr{H}(n, p)$ denote the probability space of labelled graphs on $n$ vertices, where the edges are chosen independently and at random, with probability $p$. In this case we say that the graphs are generated according to the binomial distribution of probability $p$. Though we shall not explicitely use random graphs, yet back in our mind we shall keep comparing our graphs $G_{n}$ with random graphs from $\mathscr{G}(n, p)$.

In the next theorem we shall consider properties which are trivial in case of $p$-random graphs. It is remarked in [2] that - though most of the results were considered only for the case $p=\frac{1}{2}$ - all these results generalize to every fixed probability $p \in(0,1)$. We formulate the results of [2] for arbitrary $p \in(0,1)$.

Let $G$ be a given graph and $x, y$ be two vertices of $G$. We shall denote by $S(x, y)$ the set of vertices joined to $x$ and $y$ in the same way: either to both of them or to none. Further, we shall denote by $A(x, y)$ the set of vertices joined to both $x$ and $y$. Finally, given a graph $G$, order the eigenvalues $\lambda_{i}(G)$ of its adjacency matrix so that $\left|\lambda_{i}(G)\right| \geq\left|\lambda_{i+1}(G)\right|$, for $i=1, \ldots, n-1$.

Theorem A. (Chung, Graham and Wilson, [2], [6]). Let $p \in(0,1)$ be fixed. For any graph sequence $\left(G_{n}\right)$ the following properties are equivalent:

$\mathbf{P}_{1}^{*}(\nu)$ : for fixed $\nu \geq 4$, for all graphs $L_{\nu}$

$$
\mathbf{N}^{*}\left(L_{\nu} \subseteq G_{n}\right)=(1+o(1)) n^{\nu} p^{e\left(L_{\nu}\right)}(1-p)^{\left(\begin{array}{c}
\nu \\
2
\end{array}\right)-e\left(L_{\nu}\right)}
$$

$\mathbf{P}_{2}(t):$ Let $C_{t}$ denote the cycle of length $t$. Let $t \geq 4$ be even,

$$
e\left(G_{n}\right) \geq \frac{p}{2} n^{2}+o\left(n^{2}\right) \quad \text { and } \quad \mathrm{N}\left(C_{t} \subseteq G_{n}\right) \leq(p n)^{t}+o\left(n^{t}\right)
$$


$\mathbf{P}_{3}: \quad e\left(G_{n}\right) \geq \frac{p}{2} n^{2}+o\left(n^{2}\right), \quad \lambda_{1}\left(G_{n}\right)=p n+o(n) \quad$ and $\quad \lambda_{2}\left(G_{n}\right)=o(n)$.

$\mathbf{P}_{4}: \quad$ For each subset $X \subseteq V, \quad e(X)=\frac{p}{2}|X|^{2}+o\left(n^{2}\right)$.

$\mathbf{P}_{5}:$ For each subset $X \subseteq V$, with $|X|=\left\lfloor\frac{n}{2}\right\rfloor$, we have $e(X)=\left(\frac{p}{8}+o(1)\right) n^{2}$.

$\mathbf{P}_{6}: \quad \sum_{x, y \in V}|| S(x, y)\left|-\left(p^{2}+(1-p)^{2}\right) n\right|=o\left(n^{3}\right)$.

$\mathbf{P}_{7}: \sum_{x, y \in V}|| A(x, y)\left|-p^{2} n\right|=o\left(n^{3}\right)$.

$\mathbf{P}_{8}(\alpha)$ : Fix an $\alpha \in\left(0, \frac{1}{2}\right)$. For every $X \subseteq V\left(G_{n}\right)$, with $|X|=\lfloor\alpha n\rfloor$,

$$
e\left(X, V\left(G_{n}\right)-X\right)=p \alpha(1-\alpha) n^{2}+o\left(n^{2}\right)
$$

$\left(G_{n}\right)$ is called $p$-quasi-random, if it satisfies any one (and consequently all) of the properties listed above. ${ }^{2}$

Obviously, $\mathbf{P}_{1}^{*}(\nu)$ says that the graph $G_{n}$ contains each graph of order $\nu$ with the same frequency as the random graph. Property $\mathbf{P}_{1}^{*}(\nu)$ refers to the induced copies but the analogous property for NNI copies, i.e.

$\mathbf{P}_{1}(\nu)$ : for fixed $\nu \geq 4$, for all graphs $L_{\nu}$

$$
\mathbf{N}\left(L_{\nu} \subseteq G\right)=(1+o(1)) n^{\nu} p^{e\left(L_{\nu}\right)}
$$

is equivalent with $\mathbf{P}_{1}^{*}(\nu)$.

Here $\nu \geq 4$ must be assumed: $\mathbf{P}_{1}^{*}(3)$ is not a quasi-random property. Similarly, in $\mathbf{P}_{2}(t)$ we must assume that $t \geq 4$ and is even: the analogous $\mathbf{P}_{2}(2 \ell+1)$ is neither a quasi-random property. The difference between the role of the odd and even cycles will be explained later.

As to $\mathbf{P}_{8}(\alpha), P_{8}(1 / 2)$ is an exception, again: it is not a quasi-random property.

In [13] — among others - we proved that quasi-randomness can be characterized via the Szemerédi Regularity Lemma [15], more precisely, via the Szemerédi Partition of the graph. We have formulated a graph property $\mathbf{P}_{S}$ and proved that it is equivalent with all the 8 properties $\mathbf{P}_{i}(i=1, \ldots, 8)$. The advantage of this aproach was that it made the whole "picture" fairly transparent. This characterization will be important in our approach. So we introduce the related notions and result. ${ }^{3}$

Given a graph $G$, with two disjoint subsets $X$ and $Y$ of vertices, the edgedensity between $X$ and $Y$ is defined as

$$
d(X, Y)=\frac{e(X, Y)}{|X||Y|}
$$

2 The last property can be found in [6].

3 Our result was generalized to hypergraphs by F. R. K. Chung in [4]. 
Definition 1.1. (Regularity Condition) Given a graph $G_{n}$ and two disjoint vertex sets $X \subseteq V, Y \subseteq V$, we shall call the pair $(X, Y) \quad \varepsilon$-regular, if for every $X^{*} \subset X$ and $Y^{*} \subset Y$ satisfying $\left|X^{*}\right|>\varepsilon|X|$ and $\left|Y^{*}\right|>\varepsilon|Y|$,

$$
\left|d\left(X^{*}, Y^{*}\right)-d(X, Y)\right|<\varepsilon .
$$

To formulate property $\mathbf{P}_{S}(p)$, (for a sequence $\left(G_{n}\right)$ of graphs) fix a probability $p \in(0,1)$.

$\mathbf{P}_{S}(p)$ : For every $\varepsilon>0$ and $\kappa$ there exist two integers, $k_{0}(\varepsilon, \kappa)$ and $n_{0}(\varepsilon, \kappa)$ such that, for $n>n_{0}, V\left(G_{n}\right)$ has a partition into $k$ classes $V_{1}, \ldots, V_{k}$, with $\kappa \leq k \leq$ $k_{0}(\varepsilon, \kappa),|| V_{i}|-n / k|<\varepsilon n / k$ so that for all but $\varepsilon k^{2}$ pairs $(i, j), 1 \leq i, j \leq k, i \neq j$,

$$
\left(V_{i}, V_{j}\right) \text { is } \varepsilon \text {-regular, and }\left|d\left(V_{i}, V_{j}\right)-p\right|<\varepsilon \text {. }
$$

(Mostly one assumes that $k$ may depend on $n$, though in our case, when for some fixed $p>0 d\left(V_{i}, V_{j}\right)=p+o(1)$, one can also choose a fixed $k=k_{1}(\varepsilon, \kappa)$, see [13].)

Theorem B. (Simonovits, Sós [13]). $\left(G_{n}\right)$ is p-quasi-random iff $\mathbf{P}_{S}(p)$ holds.

We will use the Szemerédi Lemma on Regular Partitions of graphs:

Regularity Lemma. (Szemerédi, [15]). For every $\varepsilon>0$ and integer $\kappa$ there exist an $n_{0}(\varepsilon, \kappa)$ and a $k_{0}(\varepsilon, \kappa)$ such that for $n>n_{0}$ for every graph $G_{n} V\left(G_{n}\right)$ can be partitioned into $k$ subsets $V_{1}, \ldots, V_{k}$ with $\kappa<k<k_{0}(\varepsilon, \kappa)$ so that ||$V_{i}|-n / k|<1$ and all but at most $\varepsilon k^{2}$ pairs $\left(V_{i}, V_{j}\right)$ are $\varepsilon$-regular.

Such partitions will be called Szemerédi Partitions. Above, ||$V_{i}|-n / k|<1$ seems to be a stronger statement than ||$V_{i}|-n / k|<\varepsilon n / k$, but using them for every $\varepsilon>0$ and $n$ sufficiently large, they are equivalent.

Komlós and Simonovits have written a long survey on the various applications of the Szemerédi Regularity Lemma [12]. The reader can find some relevant lemmas there. (For some most recent applications of the Regularity Lemma see also [11].)

\section{New results}

The main result of this paper is primarily motivated by some negative phenomena, pointed out in [2] and mentioned above. erties:

(i) $\mathbf{P}_{1}^{*}(3), \mathbf{P}_{2}(3)$, and more generally, $\mathbf{P}_{1}(2 \ell+1)$, are not quasi-random prop-

Construction 2.1. (From [2]) Let $Z_{n}$ denote a graph with $V\left(Z_{n}\right)=V_{1} \cup V_{2} \cup V_{3} \cup V_{4}$ where $V_{1}$ and $V_{2}$ span complete graphs, $V_{3}, V_{4}$ are sets of independent vertices, forming a complete bipartite graph, and each vertex of $V_{1} \cup V_{2}$ is joined to each one of $V_{3} \cup V_{4}$ independently, with probability $\frac{1}{2}$ and each vertex of $V_{3}$ is joined to each one of $V_{4}$. Let $\left|V_{i}\right|=\frac{n}{4}+o(n)$ for $i=1,2,3,4$. 
For each graph $L_{3}, Z_{n}$ contains asymptotically as many $L_{3}$ 's (and as many odd cycles $C_{2 t+1}$ for any fixed $t$ ) as a $\frac{1}{2}$-random graph, yet the graph of Construction 2.1 is very different from a $\frac{1}{2}$-random graph. (Clearly, e.g., $\mathbf{P}_{4}$ does not hold for $X=V_{1}$.)

(ii) Let $0<\alpha<\frac{1}{2} . \mathbf{P}_{8}(\alpha)$ implies that $\left(G_{n}\right)$ is a quasi-random graph sequence. However, $\mathbf{P}_{8}\left(\frac{1}{2}\right)$ is not a quasi-random property. This is shown by

Construction 2.2. (From [2]) Let $V\left(G_{n}\right)=A \cup B,|A|=|B|$. Take all the edges in $A$, none in $B$ and choose each edge joining $A$ to $B$ with probability $\frac{1}{2}$. It is easy to see that this graph satisfies the condition in (ii) with $\alpha=\frac{1}{2}$, however this is not a quasi-random graph, e.g. $\mathbf{P}_{4}$ does not hold for $X=A$, (and it is not similar at all to random graphs in $\left.\mathscr{G}\left(n, \frac{1}{2}\right)\right)$.

The reason of these negative phenomena is that the graph properties above are not hereditary in the sense that the fact that the whole graph $G_{n}$ has one of these properties does not imply that the (large) induced subgraphs of $G_{n}$ also have it.

We shall call a property hereditary if it is assumed for all the sufficiently large induced subgraphs $F_{h}$ of our graph, but (only) with the same weaker error-term $o\left(n^{\nu}\right)$.

Here we shall consider properties $\mathbf{P}$ in which we count some subgraphs of order $\nu$ in a graph $G_{n}$ and we assume that this number is the same as in the corresponding $p$-random graph with error term $o\left(n^{\nu}\right)$.

Examples of Properties.

- All induced subgraphs $F_{h}$ of $G_{n}$ have $\frac{1}{4} h^{2}+o\left(n^{2}\right)$ edges.

- All induced subgraphs $F_{h}$ of $G_{n}$ have $\frac{1}{8} h^{3}+o\left(n^{3}\right)$ labelled triangles.

- For any induced subgraph $F_{h} \subseteq G_{n}$, if $X \subseteq V\left(F_{h}\right)$ and $|X| \sim \frac{1}{2} h$, then

$$
e\left(X, V\left(F_{h}\right)-X\right) \sim \frac{1}{4} p h^{2} .
$$

The last property differs from the first two properties in that it counts not subgraphs (edges) of a subgraph, but subgraphs (edges) defined by a partition of $V\left(F_{h}\right)$.

The following assertion is fairly easy.

Theorem 2.3. Assume that for all $F_{h} \subseteq G_{n}$, for every $X \subseteq V\left(F_{h}\right)$ with $|X|=\left\lceil\frac{1}{2} h\right\rceil$

$$
e\left(X, V\left(F_{h}\right)-X\right)=\frac{p}{4} h^{2}+o\left(n^{2}\right) .
$$

Then $\left(G_{n}\right)$ is a $p$-quasi-random graph sequence. Moreover, already (1) assumed only for $h=\lceil 2 n / 3\rceil$ implies the same conclusion.

In other words, hereditarily extended $\mathbf{P}_{8}(1 / 2)$ is a quasi-random property. 
Proof. This theorem is trivial from Theorem A. Partition $V\left(G_{n}\right)$ into 3 "equal" classes $A, B, C$. Assuming (1) for $h=\lceil 2 n / 3\rceil$ we get that

$$
e\left(A, V\left(G_{n}\right)-A\right)=e(A, B)+e(A, C)=\frac{2 p}{9} n^{2}+o\left(n^{2}\right) .
$$

This is just property $\mathbf{P}_{8}(1 / 3)$. By Theorem $A$, this implies the $p$-quasirandomness.

\section{Not necessarily induced subgraphs}

Notation. Let $\nu=v(L), E=e(L)$. Denote by $\beta_{L}(p)$ and $\gamma_{L}(p)$ the "densities" of labelled induced and labelled NNI copies of $L$ in a $p$-random graph:

$$
\beta_{L}(p)=p^{E}(1-p)^{\left(\begin{array}{l}
\nu \\
2
\end{array}\right)-E} \quad \text { and } \quad \gamma_{L}(p)=p^{E} .
$$

Below we shall consider graph sequences for which not only

$$
\mathbf{N}\left(L_{\nu} \subseteq G_{n}\right)=\gamma_{L}(p) n^{\nu}+o\left(n^{\nu}\right)
$$

holds, but also for every induced subgraph $F_{h} \subseteq G_{n}$

$$
\mathbf{N}\left(L_{\nu} \subseteq F_{h}\right)=\gamma_{L}(p) h^{\nu}+o\left(n^{\nu}\right) .
$$

(Observe that in (3) we used $o\left(n^{\nu}\right)$ instead of $o\left(h^{\nu}\right)$, i.e. for small values of $h$ we allow a relatively much larger error-term. As soon as $h=o(n)$, this condition is automatically fulfilled.)

One of our main results is that for any single fixed graph $L_{\nu}$ condition (3) implies the quasi-randomness and therefore is equivalent to it.

Theorem 2.4. Let $L_{\nu}$ be a fixed sample-graph, $p \in(0,1)$ be fixed. Let $\left(G_{n}\right)$ be a sequence of graphs. If (for every sufficiently large $n$ ) for every induced $F_{h} \subseteq G_{n}$,

$$
\mathbf{N}\left(L_{\nu} \subseteq F_{h}\right)=\gamma_{L}(p) h^{\nu}+o\left(n^{\nu}\right)
$$

then $\left(G_{n}\right)$ is p-quasi-random.

The main points of this result (compared to the earlier results) are that

(a) $\mathbf{P}_{1}(\nu)$ assumed hereditarily implies quasi-randomness for $\nu=3$ as well; and

(b) our assumption refers to a single graph $L_{\nu}$ instead of assuming the condition for all the graphs on $\nu$ vertices (as in $\mathbf{P}_{1}(\nu)$ )

\section{However}

(c) In Theorem A it is the same if we count induced or NNI copies of $L_{\nu}$. Here the two cases are not equivalent (since we count only a single graph $L_{\nu}$ ). The case of induced subgraphs is much more involved and the corresponding implication does not always hold: the formula analogous to (4), but assumed for the induced subgraphs (namely (5) below) does not necessarily imply the quasi-randomness of $\left(G_{n}\right)$. 


\section{Induced subgraphs}

Next we consider how Theorem 2.4 can be extended to "Induced Copies". One would like to know if for given $\left(L_{\nu}, p\right)$ the following is true or not:

"Property $\mathscr{H}\left(L_{\nu}, p\right)$ ". Given a sample graph $L_{\nu}$ and a probability $p$, if for a graph sequence $\left(G_{n}\right)$ for every induced subgraph $F_{h}$ of $G_{n}$

$$
\mathbf{N}^{*}\left(L_{\nu} \subseteq F_{h}\right)=\beta_{L}(p) h^{\nu}+o\left(n^{\nu}\right)
$$

then $\left(G_{n}\right)$ is p-quasi-random

Property $\mathscr{H}\left(L_{\nu}, p\right)$ is mostly false in this form, for two reasons, both being algebraic coincidences. For some graphs $L$ it may happen that fixing two groups $V_{1}$ and $V_{2}$ of vertices and joining any pair $(x, y)$ independently, with probability $p$ if $x, y \in V_{1}$, with probability $q$ if $x, y \in V_{2}$ and with probability $s$ if $x \in V_{1}, \quad y \in V_{2}$ we get a sequence of counterexamples. Here we do not go into detailed discussion of this phenomenon, rather we state one counterexample and return to the detailed analysis of this question in [14].

Construction 2.5. Let $V\left(G_{n}\right)=V_{1} \cup V_{2}$, join the pairs in $V_{1}$ with probability $p$, in $V_{2}$ with probability $q$ and between them with probability $s$. For every $p>\frac{1}{\sqrt{3}}$ there exists an $s \in(0,1)$ such that for $q:=p$ the resulting sequence is a counterexample for Property $\mathscr{H}\left(P_{3}, p\right)$. In particular, for

$$
\begin{aligned}
& p=q=\frac{4}{5} \text { choose } s=\frac{12}{35}, \text { for } \\
& p=q=\frac{1}{\sqrt{3}} \text { choose } s=1 .
\end{aligned}
$$

Some results concerning the induced case and the detailed analysis and calculations concerning this counterexample can be found in [14].

\section{Tools to prove our theorems}

Here we will count small subgraphs $L_{\nu}$ of $G_{n}$, spanned by a fixed number (namely by $(\nu-1)$ ) of classes of the Szemerédi Partition. In this case the number of $L_{\nu}$ 's also depends noticeably on the number of edges within the classes. The result we need (Lemma 3.1 below) is a variant of the following.

Definition. Given an $r \times r$ matrix $D=\left(d_{i, j}\right)$, and $r$ disjoint sets of vertices, $V_{1}, \ldots, V_{r}$, we define the corresponding generalized random graph as follows: for each $x \in V_{i}$, $y \in V_{j}, x \neq y,(1 \leq i \leq j \leq r)$ we join $x$ and $y$ independently, at random, with probability $d_{i, j}$.

Theorem C. ([13]) For a given $\delta$ and $a \kappa>\frac{1}{\delta}$, let $\left\{V_{1}, \ldots, V_{k}\right\}$ be a Szemerédi Partition of an arbitrary graph $G_{n}$, corresponding to the parameters $\varepsilon=\delta^{2}, \kappa$. Let $Q_{n}$ be a $k$-partite generalized random graph obtained by replacing the edges 
joining the classes $V_{i}$ and $V_{j}$ by independently chosen random edges of probability $d_{i, j}:=d\left(V_{i}, V_{j}\right),(1 \leq i<j \leq k)$, and deleting the edges in $G\left[V_{i}\right], i=1, \ldots, k$. (Set $d_{i, i}=0$.) Then, for $n>n_{0}(\delta, \kappa)$,

$$
\left|\mathbf{N}\left(L_{\nu} \subseteq Q_{n}\right)-\mathbf{N}\left(L_{\nu} \subseteq G\right)\right| \leq C_{\nu} \delta n^{\nu}
$$

almost surely ${ }^{4}$ where $C_{\nu}$ is a constant depending only on $\nu$,

To formulate the version of the above theorem used in our proofs, we need the following.

Notation. Given a graph $G$ the vertices of which are partitioned into $\nu-1$ classes, $U_{1}, \ldots, U_{\nu-1}$, let $\mathbf{N}_{f}\left(L_{\nu} \subseteq G\right)$ denote the number of those labelled NNI copies of $L_{\nu}$ which meet all the classes $U_{i}, 1 \leq i<j \leq \nu-1$. (The " $f$ " refers to the full size.)

Lemma 3.1. Let $G[U]$ be a graph with vertex set $U$ partitioned into $U_{1}, \ldots, U_{\nu^{\prime}-1}$. Suppose the pairs $\left(U_{i}, U_{j}\right)$ are $\varepsilon$-regular for $1 \leq i<j \leq \nu-1$. Let $Q[U]$ be the graph obtained by joining each $x \in U_{i}$ to each $y \in U_{j}$ at random, independently, with probability $d_{i, j}:=d\left(U_{i}, U_{j}\right)$ for every $1 \leq i<j<\nu$. Let us keep the original edges of $G\left[U_{i}\right]$ 's (i.e. the edges within the classes) unchanged in $Q[U]$. Then, almost surely, for some constant $c_{\nu}$, (depending only on $\nu$ )

$$
\left|\mathbf{N}_{f}\left(L_{\nu} \subseteq G[U]\right)-\mathbf{N}_{f}\left(L_{\nu} \subseteq Q[U]\right)\right|<c_{\nu} \varepsilon|U|^{\nu} .
$$

As a matter of fact, we shall regard 3 graphs: $G[U], Q[U]$ defined above and $Q^{*}[U]$ which is obtained by replacing the edges within the classes $U_{i}$ also by randomly, independently chosen edges of probabiltity $d_{i, i}$. In other words, we join the vertices $x \in U_{i}$ and $y \in U_{j}$ with edge-probability $d_{i, j}$ for $1 \leq i \leq j \leq \nu-1$ (unless $x=y$ ).

Remark. In Lemma 3.1 the edges within the classes $V_{i}$ cannot be neglected. Actually this is good for us: this enables us to count them. There is an other case where one uses edges within the partition classes: in Ramsey-Turán type theorems, (see e.g. [16]). The Turán-Ramsey theorems show that (in some sense) the limit of validity of the above lemma is when we allow just one edge within just one partition class.

This lemma is equally valid for induced and NNI, labelled and unlabelled copies. The proof is essentially the same in all the four cases, (or one can derive the induced version from the noninduced one and vice versa). Since here we shall use the NNI version, we formulate the proof for that case. In the graph $Q[U]$ one can easily calculate the number of copies of $L_{\nu}$ 's.

Below we formulate a variant of Lemma 3.1, called the Main Lemma, where, instead of saying that the graph and its randomlike approximation have roughly

4 Here the "almost surely" refers to the random graph $Q_{n}$ constructed to approximate $G_{n}$. Clearly, $G_{n}$ does not depend on any random choice. 
the same number of $L_{\nu}$ 's, we provide this number. We shall use - and therefore we shall prove - below only the Main Lemma. Yet Lemma 3.1 is the one showing the real meaning of the Main Lemma.

Main Lemma. Let $G[U]$ be a graph with vertex set $U$ partitioned into $U_{1}, \ldots, U_{\nu-1}$. Suppose the pairs $\left(U_{i}, U_{j}\right)$ are $\varepsilon$-regular for $1 \leq i<j \leq \nu-1$. Then

$$
\mathbf{N}_{f}\left(L_{\nu} \subseteq G[U]\right)=\sum_{i=1}^{\nu-1}\left(A_{i} \frac{e\left(U_{i}\right)}{\left|U_{i}\right|}+B_{i}\left|U_{i}\right|\right) \prod_{j=1}^{\nu-1}\left|U_{j}\right|+O(\varepsilon|U|) \prod_{j=1}^{\nu-1}\left|U_{j}\right|
$$

where $A_{i}$ and $B_{i}$ depend only on $L_{\nu}$ and on the densities $d_{i, j}=d\left(U_{i}, U_{j}\right)$ for $1 \leq i<j \leq \nu-1$, and the constant in $O($.$) depends only on \nu$.

In fact, we will use only the following corollary of the Main Lemma:

Corollary 3.2. Let $V\left(G_{n}\right)=V_{1} \cup \ldots \cup V_{k}$ be an $\varepsilon$-regular partition, $\left|V_{i}\right| \sim m=\lfloor n / k\rfloor$, $1 \leq i \leq k$. Suppose that $\left(V_{i}, V_{j}\right)$ are all $\varepsilon$-regular for $1 \leq i<j \leq \nu-1$. Let $X \subseteq V_{1}$, ||$X\left|-\frac{m}{2}\right|<\varepsilon m$. Let $G_{X}:=G\left[X \cup V_{2} \cup \ldots \cup V_{\nu-1}\right]$. Then

$$
\mathrm{N}_{f}\left(L_{\nu} \subseteq G_{X}\right)=A e(X) m^{\nu-2}+B m^{\nu}+O(\varepsilon) m^{\nu},
$$

where $A$ and $B$ depend only on the densities $d_{i, j}=d\left(V_{i}, V_{j}\right), 1 \leq i<j \leq \nu-1$ and on $d_{i, i}=2 e\left(V_{i}\right) / m^{2}$ for $2 \leq i \leq \nu-1$.

\section{Proof of the Main Lemma}

We will use the following simple consequence of the $\varepsilon$-regularity.

Proposition 3.3. Let $\left(U_{1}, U_{2}\right)$ be an $\varepsilon$-regular pair, $W \subseteq U_{2}$ with $|W|>\varepsilon\left|U_{2}\right|$ and let $d_{1,2}:=d\left(U_{1}, U_{2}\right)$. Let $Z \subset U_{1}$ be defined by

$$
Z=\left\{x: x \in U_{1},|N(x) \cap W|<\left(d_{1,2}-\varepsilon\right)|W|\right\} .
$$

Then $|Z|<\varepsilon\left|U_{1}\right|$.

Proof. Clearly, $e(Z, W)<\left(d_{1,2}-\varepsilon\right)|Z||W|$, i.e. $d(Z, W)<d_{1,2}-\varepsilon$. Hence, by the $\varepsilon$-regularity, $|Z|<\varepsilon\left|U_{1}\right|$.

A labelled NNI copy $H_{\nu}$ of $L_{\nu}$ in $G[U]$ is given by a mapping $\psi: V\left(L_{\nu}\right) \rightarrow U$. Let $V\left(L_{\nu}\right)=\left\{x_{0}, \ldots, x_{\nu-1}\right\}$ and $y_{i}=\psi\left(x_{i}\right)$ for $0 \leq i \leq \nu-1$. We define the position of such an $H_{\nu} \subseteq G[U]$ as follows.

Definition. Let $\Phi:\{0, \ldots, \nu-1\} \rightarrow\{1, \ldots, \nu-1\}$ be a mapping. We say that $\left(y_{0}, \ldots, y_{\nu-1}\right)$ has position $\Phi$, if $y_{i} \in U_{\Phi(i)}$ for $0 \leq i \leq \nu-1$. A position is called full if it meets all the $\nu-1$ classes $U_{i}$. The full positions are of type (a) and (b) according 
to whether the two vertices of $L_{\nu}$ mapped into the same class $U_{i}$ are independent in $L_{\nu}$ or joined.

Remember that a "copy" $H_{\nu} \subseteq G$ of $L_{\nu}$ is an ordered $\nu$-tuple $\left(y_{0}, \ldots, y_{\nu-1}\right) \subset U$ if for every $\left(x_{i}, x_{j}\right) \in E\left(L_{\nu}\right),\left(y_{i}, y_{j}\right)$ is an edge of $G$.

We count the number of "copies" $H_{\nu} \subseteq G[U]$ i.e. sequences $\left(y_{0}, \ldots, y_{\nu-1}\right) \subset U$ according to their position $\Phi$. Then we sum up these numbers for the positions. Let $\mathbf{N}_{f}\left(L_{\nu} \subseteq G[U] \mid \Phi\right)$ denote the number of "copies" of the (full) position $\Phi$. By symmetry we may restrict ourselves to the case when $\Phi(0)=1$ and $\Phi(i)=i$ for $1 \leq i \leq \nu-1$. Let $\Phi_{0}$ denote this position.

The proof is mostly standard, the same as the proof of Theorem $\mathrm{C}$ in [13]. Namely, we think of $G[U]$ as if it were a generalized random graph, $Q^{*}[U]$, where $Q^{*}[U]$ is obtained from $Q[U]$ by randomizing the edges within the classes as well; we count the number of copies of $L_{\nu}$ meeting all the sets $U_{i}(1 \leq i \leq \nu-1)$ in $Q[U]$, and make the same calculations for $G[U]$, always using the regularity of pairs $\left(U_{i}, U_{j}\right)$ and that the regularity implies that these numbers are roughly the same for our graphs $G[U], Q[U]$ and $Q^{*}[U]$.

First we consider the generalized random graph $Q[u]$. Let $\widetilde{R}_{Q}\left(L_{\nu} ; \Phi_{0}\right)$ denote the expected value of the "copies" of $L_{\nu}$ of position $\Phi_{0}$, in $Q^{*}[U]$. Then it is easy to see that if

$$
\prod^{*}:=\prod_{\substack{\left(x_{i}, x_{j}\right) \in E\left(L_{\nu}\right) \\ 1 \leq i<j \leq \nu-1}}
$$

then

$$
\widetilde{R}_{Q}\left(L_{\nu} ; \Phi_{0}\right)= \begin{cases}d_{1,1}\left|U_{1}\right| \prod_{t=1}^{\nu-1}\left|U_{t}\right| \prod^{*} d_{i, j} & \text { if }\left(x_{0}, x_{1}\right) \in E\left(L_{\nu}\right) \\ \left|U_{1}\right| \prod_{t=1}^{\nu-1}\left|U_{t}\right| \prod^{*} d_{i, j} & \text { otherwise. }\end{cases}
$$

Summing (7) for all the positions $\Phi$ we get that

$$
\sum_{\Phi} \widetilde{R}_{Q}\left(L_{\nu} ; \Phi\right)=\sum_{i} A_{i} \frac{e\left(U_{i}\right)}{\left|U_{i}\right|} \prod_{j=1}^{\nu-1}\left|U_{j}\right|+\sum_{i} B_{i}\left|U_{i}\right| \prod_{j=1}^{\nu-1}\left|U_{j}\right|
$$

where $A_{i}$ and $B_{i}$ are constants depending only on the densities $d_{i j}$ : this formula is just the one corresponding to (6) in the Main Lemma.

To build up (many) copies of $L_{\nu}$ in $G[U]$, with the given Szemerédi Partition, $U_{1}, \ldots, U_{k}$, we will build an $L_{\nu}$ with vertices $y_{0}, y_{1} \in U_{1}, \ldots, y_{\nu-1} \in U_{\nu-1}$, step by step, recursively. For this we need some notation and definitions.

Definition. If $y_{0}, \ldots, y_{t-1}$ have been fixed arbitrarily but so that

$$
y_{0}, y_{1} \in U_{1}, \ldots, y_{\ell} \in U_{\ell}, \ldots, y_{t-1} \in U_{t-1},
$$


then let for $j \geq t$

$$
W_{t, j}=W_{t, j}\left(y_{0}, \ldots, y_{t-1}\right):=U_{j} \bigcap\left(\bigcap_{\substack{\left(x_{i}, x_{j}\right) \in E\left(L_{\nu}\right) \\ 0 \leq i<t}} N\left(y_{i}\right)\right) .
$$

For $j<t$, i.e. having selected $y_{j}$, we put $W_{t, j}:=\left\{y_{j}\right\}$. Obviously

$$
U_{j} \supseteq W_{0, j} \supseteq W_{1, j} \supseteq \ldots \supseteq W_{t-1, j} \supseteq W_{t, j} .
$$

Now we define a subset $Y_{t} \subseteq W_{t, t}: Y_{t}$ is the set of $y \in W_{t, t}$ for which (for $j \geq t+1$ )

$$
\left|W_{t+1, j}\left(y_{0}, \ldots, y_{t-1}, y\right)\right| \geq\left(d_{t, j}-\varepsilon\right)\left|W_{t, j}\right| \geq\left(\prod_{\substack{\left(x_{i}, x_{j}\right) \in E\left(L_{\nu}\right) \\ 0 \leq i \leq t}}\left(d_{i, j}-\varepsilon\right)\right)\left|U_{j}\right|
$$

The definition of $Y_{t}$ deserves some explanation. When - having fixed $y_{0}, y_{1}, \ldots, y_{t-1}$ and starting $\operatorname{Step}(\mathrm{t})$ to fix the image $y_{t}$ of $x_{t} \in L_{\nu}$ our previous choices restrict us to $W_{t, t} \subseteq U_{t}$. Further, our later choices are restricted to some subsets $W_{t, j} \subseteq U_{j}, j>t$. So in Step(t), fixing $y_{t}$ we do not wish to spoil our later possibilities too much by decreasing some $W_{t+1, j}$ too much: we use the regularity to guarantee that $\left|W_{t+1, j}\right|$ remains large for each $j>t$ : we shall choose only such $y \in W_{t, t}$ for which (for every $j \geq t+1$ ) (8) holds. The set of these $y \in W_{t, t}$ will be denoted by $Y_{t}$. Below (summing the contributions of various positions) we shall discard all those positions where one of the sets $W_{t, j}$ may become smaller than $\varepsilon|U|$. In the remaining cases, by Proposition 3.3,

$$
\left|Y_{t}\right|>\left|W_{t, t}\right|-\nu \varepsilon\left|U_{t}\right|
$$

We shall assume in all our proofs that $\varepsilon<\frac{1}{10 \nu}$.

There are only $\nu$ ! positions of $L_{\nu}$, and if for some position $\Phi$ the corresponding number

$$
\widetilde{R}_{Q}\left(L_{\nu} ; \Phi\right)<2 \nu \varepsilon|U| \prod_{j=1}^{\nu-1}\left|U_{j}\right|
$$

then this position can be forgotten: its contribution is swallowed by the error-term $O\left(\varepsilon|U| \prod\left|U_{j}\right|\right)$ of (6). This will imply below that all the sets $\left|W_{t, j}\right|>2 \nu \varepsilon|U|$ for $j \geq t$. Therefore below we shall always be allowed to use Proposition 3.3.

To prove (6) first we prove a lower bound for $\mathbf{N}_{f}\left(L_{\nu} \subseteq G[U] \mid \Phi_{0}\right)$.

To build up the "copies" of $L_{\nu}$ of position $\Phi_{0}$, we start with $W_{0, j}:=U_{j}$ for $j=1, \ldots, \nu-1$. In $\operatorname{Step}(0)$ and $\operatorname{Step}(1)$ we pick the vertices $y_{0}, y_{1} \in U_{1}$. In $\operatorname{Step}(\mathbf{t})$ 
$\left(t>1\right.$ ) we have already fixed $y_{0}, y_{1} \in U_{1}, y_{j} \in U_{j}$ for $j<t$, some sets $W_{t, j} \subset U_{j}$ (as possible choices of $y_{j}: j \geq t$ ) and we fix $y_{t} \in W_{t, t} \subseteq U_{t}$ so that (8) remains valid.

Step(0). (Choosing y0.) Let us define

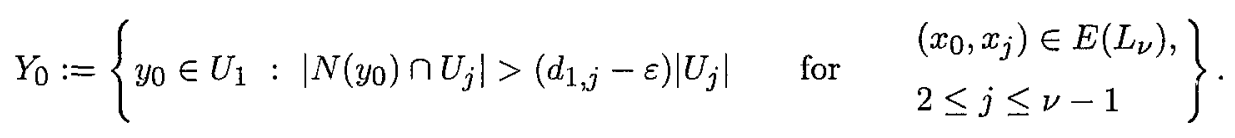

By the $\varepsilon$-regularity and by Propositon 3.3

$$
\left|Y_{0}\right|>\left|U_{1}\right|-\nu \varepsilon\left|U_{1}\right|
$$

For a fixed $y_{0} \in Y_{0}$, for $j=2, \ldots, \nu-1$ define the sets

$$
W_{0, j}\left(y_{0}\right):= \begin{cases}N\left(y_{0}\right) \cap U_{j} & \text { if }\left(x_{0}, x_{j}\right) \in E\left(L_{\nu}\right) \\ U_{j} & \text { otherwise. }\end{cases}
$$

$\operatorname{Step}(1 / \mathbf{a})$. (Choosing $y_{1}$.) If $\left(x_{0}, x_{1}\right) \in E\left(L_{\nu}\right)$, put

$$
\begin{aligned}
Y_{1}\left(y_{0}\right):=\left\{y_{1} \in U_{1} \cap N\left(y_{0}\right):\right. & \left|N\left(y_{1}\right) \cap W_{0, j}\left(y_{0}\right)\right|>\left(d_{1, j}-\varepsilon\right)\left|W_{0, j}\left(y_{0}\right)\right| \\
& \text { for } \left.\quad\left(x_{1}, x_{j}\right) \in E\left(L_{\nu}\right), \quad 2 \leq j \leq \nu-1\right\} .
\end{aligned}
$$

and define for $y_{1} \in Y_{1}\left(y_{0}\right)$ and for $j=2, \ldots, \nu-1$,

$$
W_{1, j}\left(y_{0}, y_{1}\right):= \begin{cases}N\left(y_{1}\right) \cap W_{0, j}\left(y_{0}\right) & \text { if }\left(x_{1}, x_{j}\right) \in E\left(L_{\nu}\right) \\ W_{0, j}\left(y_{0}\right) & \text { otherwise. }\end{cases}
$$

Assume that $y_{0}$ is fixed and its degree is $d\left(y_{0}\right)$ in $G\left[U_{1}\right]$. We can try to choose any neighbor $y_{1}$ and at most $\nu \varepsilon\left|U_{1}\right|$ are ruled out by the fact that they "ruin the future". So

$$
\sum_{y_{0} \in Y_{0}} d\left(y_{0}\right)>2 e\left(U_{1}\right)-\nu \varepsilon\left|U_{1}\right|^{2}
$$

and the number of pairs $\left(y_{0}, y_{1}\right)$ to be chosen is at least

$$
\sum_{y_{0} \in Y_{0}}\left(d\left(y_{0}\right)-\nu \varepsilon\left|U_{1}\right|\right)>2 e\left(U_{1}\right)-2 \nu \varepsilon\left|U_{1}\right|^{2}
$$

$\operatorname{Step}(\mathbf{1} / \mathbf{b})$. If $\left(x_{0}, x_{1}\right) \notin E\left(L_{\nu}\right)$, then the restriction on $\left(y_{0}, y_{1}\right)$ that it must be an edge of $G\left[U_{1}\right]$ can be forgotten: the above argument works word by word if we replace $G\left[U_{1}\right]$ by a complete graph: $\left|Y_{1}\left(y_{0}\right)\right|>\left|U_{1}\right|-\nu \varepsilon\left|U_{1}\right|$ and $y_{0}, y_{1} \in U_{1}$ can be chosen in at least

$$
(1-2 \nu \varepsilon)\left|U_{1}\right|^{2}
$$

ways, maintaining large $W_{1, j}$ 's. 
$\operatorname{Step}(\mathbf{t})$. Suppose we have already fixed the vertices $y_{0}, y_{1} \in U_{1}, \ldots, y_{t-1} \in U_{t-1}$. For $t \leq j \leq \nu-1$ let $W_{t, j}$ be the set of possible choices of $y_{j} \in U_{j}$ after the first $t$ vertices, $y_{0}, \ldots, y_{t-1}$ have been fixed and we set out to find $y_{t} \in W_{t, t}$.

Let for $y \in W_{t, t} \subseteq U_{t}$, and $j>t^{5}$

$$
W_{t+1, j}(y):= \begin{cases}N(y) \cap W_{t, j} & \text { if }\left(x_{t}, x_{j}\right) \in E\left(L_{\nu}\right) \\ W_{t, j} & \text { otherwise. }\end{cases}
$$

Now, as in $\operatorname{Step}(0)$ and $\operatorname{Step}(1)$, we keep only those points $y \in W_{t, t}$ for which, whenever $j>t$ and $\left(x_{t}, x_{j}\right) \in E\left(L_{\nu}\right)$, then

$$
\left|W_{t+1, j}(y)\right|=\left|N(y) \cap W_{t, j}\right|>\left(d_{t, j}-\varepsilon\right)\left|W_{t, j}\right|
$$

Put

$$
\underline{Y}_{t}=\left\{\begin{array}{l}
y: y \in W_{t, t} \quad \text { and (12) holds for all } \begin{array}{l}
t<j \leq \nu-1 \\
\left(x_{t}, x_{j}\right) \in E\left(L_{\nu}\right)
\end{array} \text { and }
\end{array}\right\} .
$$

By the construction,

$$
\left|W_{t, t}\right|>\left|U_{t}\right| \cdot \prod_{\substack{\left(x_{j}, x_{t}\right) \in E\left(L_{\nu}\right) \\ 0 \leq j<t}}\left(d_{t, j}-\varepsilon\right)
$$

and by the $\varepsilon$-regularity

$$
\left|Y_{t}\right|>\left|W_{t, t}\right|-\nu \varepsilon\left|U_{t}\right|>\left|U_{t}\right|\left(\prod_{\substack{\left(x_{j}, x_{t}\right) \in E\left(L_{\nu}\right) \\ 0 \leq j<t}}\left(d_{t, j}-\varepsilon\right)-\nu \varepsilon\right) .
$$

- If $\left(x_{0}, x_{1}\right) \in E\left(L_{\nu}\right)$, then we get that

$$
\begin{gathered}
\mathbf{N}_{f}\left(L_{\nu} \subseteq G[U] \mid \Phi_{0}\right) \geq\left(2 e\left(U_{1}\right)-2 \nu \varepsilon\left|U_{1}\right|^{2}\right) \prod_{t=2}^{\nu-1}\left|Y_{t}\right| \\
\geq\left(2 e\left(U_{1}\right)-2 \nu \varepsilon\left|U_{1}\right|^{2}\right)\left(\prod_{t=2}^{\nu-1}\left|U_{t}\right|\right)\left(\prod_{t=2}^{\nu-1}\left(\prod_{\substack{\left.x_{j}, x_{t}\right) \in E\left(L_{\nu}\right) \\
0 \leq j<t}}\left(d_{i, j}-\varepsilon\right)\right)-\nu \varepsilon\right) \\
=2 e\left(U_{1}\right) \prod_{t=2}^{\nu-1}\left|U_{t}\right| \prod^{\mathrm{s}} d_{i, j}-O\left(\varepsilon\left|U_{1}\right| \prod_{t=1}^{\nu-1}\left|U_{t}\right|\right) .
\end{gathered}
$$

5 Below we abbreviate to $W_{i, j}(y)$ and $W_{i, j}$ what normally would be $W_{i, j}\left(y_{0}, y_{1}, \ldots, y_{t-1}, y\right)$ and $W_{i, j}\left(y_{0}, y_{1}, \ldots, y_{t-1}\right)$, respectively. 
- If $\left(x_{0}, x_{1}\right) \notin E\left(L_{\nu}\right)$, then we replace in the above formulas $2 e\left(U_{1}\right)$ by $\left|U_{1}\right|^{2}$.

$$
\begin{aligned}
\mathbf{N}_{f}\left(L_{\nu} \subseteq G[U] \mid \Phi_{0}\right) & \geq\left(\left|U_{1}\right|^{2}-2 \nu \varepsilon\left|U_{1}\right|^{2}\right) \prod_{t=2}^{\nu-1}\left|Y_{t}\right| \\
& =\left|U_{1}\right|^{2} \prod_{t=2}^{\nu-1}\left|U_{t}\right| \prod^{*} d_{i, j}-O\left(\varepsilon\left|U_{1}\right| \prod_{t=1}^{\nu-1}\left|U_{t}\right|\right)
\end{aligned}
$$

In a similar way we can get the upper bound for $\mathrm{N}_{f}\left(L_{\nu} \subseteq G[U] \mid \Phi_{0}\right)$. Hence

$$
\begin{aligned}
\mathbf{N}_{f}\left(L_{\nu} \subseteq G[U] \mid \Phi_{0}\right)= \\
\qquad \begin{cases}\left(2 e\left(U_{\jmath}\right)+O(\varepsilon)\right)\left(\prod_{t=2}^{\nu-1}\left|U_{t}\right|\right)\left(\prod^{*} d_{i, j}\right) & \text { if }\left(x_{0}, x_{1}\right) \in E\left(L_{\nu}\right), \\
(1+O(\varepsilon))\left|U_{1}\right|^{2}\left(\prod_{l=2}^{\nu-1}\left|U_{t}\right|\right)\left(\prod^{*} d_{i, j}\right) & \text { otherwise. }\end{cases}
\end{aligned}
$$

Summing the corresponding formulas for all the positions we get the assertion of the Main Lemma,

\section{Proof of Theorem 2.4}

To start with, we remind the reader that the main condition of Theorem 2.4 is that:

(*) $\quad \mathrm{N}\left(L_{\nu} \subseteq F_{h}\right)=\gamma_{L}(p) h^{\nu}+o\left(n^{\nu}\right) \quad$ for every induced $\quad F_{h} \subseteq G_{n}$.

\section{The main idea}

The idea is fairly transparent. We shall show that if $\left(G_{n}\right)$ satisfies (*) and $\left\{V_{1}, \ldots, V_{k}\right\}$ is a Szemerédi partition of $V\left(G_{n}\right)$, while $\varepsilon \rightarrow 0$ very slowly and $n \rightarrow \infty$, then

(i) most of the graphs $G\left[V_{\hat{i}}\right](i=1,2, \ldots, k)$ are $p$-quasi-random. (Here we apply the Main Lemma to $v-1$ groups $V_{i}$.)

(ij) Using (i) and applying condition (*) to the graphs $F_{h}=G\left[V_{i} \cup V_{j}\right]$ we shall show that almost all densities $1 \leq i, j \leq k d\left(V_{i}, V_{j}\right)=p+o(1)$.

We emphasize again that mostly, using the Szemerédi Regularity Lemma one does not care for the edges within the classes: their number is negligibie. The reason why here we care is that in a crucial step we consider $G\left[V_{i} \cup V_{j}\right]$ where $e\left(G\left[V_{i}\right]\right)$ is not negligible anymore, and it is good for us here that these edges do matter.

Proof of Theorem 2.4. Let $\left(G_{n}\right)$ be a sequence satisfying $(*)$. We apply the Szemerédi Lemma to $\left(G_{n}\right)$ with $\varepsilon_{n} \rightarrow 0$ sufficiently slowly. 
Let $\tilde{\varepsilon}_{t}=\frac{1}{t}, \tilde{\kappa}_{t}=t$. Let $k_{0}:=k_{0}\left(\frac{1}{t}, t\right), n_{t}^{*}:=n^{*}\left(\frac{1}{t} ; t\right)$ be the thresholds of the Regularity Lemma belonging to these parameters.

The assumption (*) in Theorem 2.4 means that for $F_{h} \subseteq G_{n}$

$$
\left|\mathbf{N}\left(L_{\nu} \subseteq F_{h}\right)-\gamma_{L}(p) \cdot h^{\nu}\right|<\delta_{n} \cdot n^{\nu}
$$

for some sequence $\delta_{n} \rightarrow 0$ if $n \rightarrow \infty$. We define $n_{t}^{* *} \rightarrow \infty$ by

$$
\delta_{n} \cdot k_{0}\left(\frac{1}{t}, t\right)^{\nu}<\frac{1}{t} \quad \text { for } \quad n>n_{t}^{* *} .
$$

Put $n_{t}=\max \left(n_{t}^{*}, n_{t}^{* *}, n_{t-1}+1\right)$. Now, for each $n$ we fix that very $t=t(n)$ for which $n_{t}<n \leq n_{t+1}$. Fix for each $G_{n}$ a Szemerédi Partition $V\left(G_{n}\right)=V_{1}^{n} \cup \ldots \cup V_{k(n)}^{n}$ with the corresponding parameters $\tilde{\varepsilon}_{t}$ and $\tilde{\kappa}_{t}=t$. Then the above partitions are just the Szemerédi-Partitions of $G_{n}$ belonging to the parameters $\varepsilon_{n}=\frac{1}{t}$ and $\kappa_{n}=t$. For the corresponding $k(n)$ we have $t<k(n)<k_{0}\left(\frac{1}{t}, t\right)$.

Observe that by (13) and (14) we have for every induced $F_{h} \subseteq G_{n}, n_{t}<n \leq n_{t+1}$

$$
\left|\mathbf{N}\left(L_{\nu} \subseteq F_{h}\right)-\gamma_{L}(p) \cdot h^{\nu}\right|<\tilde{\varepsilon}_{t} h^{\nu}=\varepsilon_{n} h^{\nu}
$$

if $h>\frac{n}{k(n)}$.

We will prove the theorem by proving the following two lemmas:

Lemma 4.1. Under the conditions of Theorem 2.4, using the partitions as described above, for all but $o(k(n))$ choices of $i(n), 1 \leq i(n) \leq k(n)$, the graph sequence $\left(G_{n}\left[V_{i(n)}^{n}\right]\right)$ is a $p$-quasi random graph sequence.

Lemma 4.1 is used only to prove

Lemma 4.2. Under the conditions of Theorem 2.4, using the partitions as described above, for all but $o\left(k(n)^{2}\right)$ choices of $(i(n), j(n)), 1 \leq i(n)<j(n) \leq k(n),\left(\leq k_{0}(1 / t, t)\right)$ for the densities $d_{i(n), j(n)}:=d\left(V_{i(n)}^{n}, V_{j(n)}^{n}\right)$

$$
d_{i(n), j(n)} \longrightarrow p \quad \text { for } \quad n \rightarrow \infty
$$

How to prove Theorem 2.4 using Lemma 4.2? By Theorem B, Lemma 4.2 implies that the sequence $\left(G_{n}\right)$ is p-quasi-random. Of course, in Theorem B we require that for every fixed $\varepsilon$ there be an appropriate Szemerédi partition with at most $K(\varepsilon)$ classes, with almost all densities around $p$, and here we have a sequence of Szemerédi partitions, where the number of classes tends to infinity and $\varepsilon \rightarrow 0$. So, formally the conclusion of the above lemmas is not the same as $\mathbf{P}_{S}(p)$. Yet, they are equivalent, and we need only that the conclusion of Lemma 4.2 implies $\mathbf{P}_{S}(p)$.

Namely, for fixed $\varepsilon>0$ and $\kappa>0$ let us fix

$$
K=\max \left\{\frac{1}{2 \varepsilon}, \kappa\right\} .
$$


Take a random partition of the classes $\left\{V_{i(n)}^{n}\right\}$, of Lemma 4.2 into $K$ roughly equal families of classes, $\mathscr{Z}_{1}, \ldots, \mathscr{Z}_{K}$, and let for $\ell=1, \ldots, K$,

$$
W_{\ell}:=\bigcup_{V_{i(n)}^{n} \in \mathscr{L}_{\ell}} V_{i(n)}^{n}
$$

be the corresponding $K$-partitioning of $V\left(G_{n}\right)$. Taking a random partition we have achieved that the non-regular pairs of classes are uniformly spread. Thus, clearly, $d\left(W_{\ell}, W_{\ell^{\prime}}\right) \rightarrow p$ (uniformly) as $n \rightarrow \infty$. So $\left(G_{n}\right)$ satisfies $P_{S}(p)$. Thus Lemma 4.2 implies the $p$-quasi-randomness. ${ }^{6}$

Proof of Lemma 4.1. Given an $\varepsilon>0$ and a Szemerédi Partition of $V\left(G_{n}\right)$ into $V_{1}, \ldots, V_{k}$, we shall call the $(\nu-1)$-tuple $V_{i_{1}}, \ldots, V_{i_{\nu-1}}$ good if all the pairs $\left(V_{i_{a}}, V_{i_{b}}\right)$ are $\varepsilon$-regular $(1 \leq a<b \leq \nu-1) \cdot{ }^{\top}$ A group $V_{i}$ is good if it is contained in a good $(\nu-1)$-tuple.

Let $F_{k}$ denote the graph the vertices of which are $V_{1}, \ldots, V_{k}$ and the edges are those $\left(V_{a}, V_{b}\right)$ 's which are $\varepsilon$-regular. Since $F_{k}$ is almost a $K_{k}$ : at most $\varepsilon k^{2}$ edges are missing, therefore we may delete at most $5 \varepsilon \nu k$ vertices so that in the remaining $F_{k^{\prime}}^{*}$ all the vertices have degree at least $\left(1-\frac{1}{2 y}\right) k^{\prime}$. But a greedy building-up algorithm shows that each edge (and of course each vertex) of $V\left(F_{k^{\prime}}^{*}\right)$ is contained in a $K_{\nu}$.

So at least $k-5 \nu \varepsilon k$ of the groups are good and all but at most $5 \nu \varepsilon k^{2}$ of the pairs are covered by good $(\nu-1)$-tuples.

Let $n_{t}<n \leq n_{t+1}$ and $V\left(G_{n}\right)=V_{1}^{(n)} \cup \ldots \cup V_{k}^{(n)}$ be a Szemerédi Partition of $G_{n}$ with the parameters $\tilde{\varepsilon}_{t}=\frac{1}{t}, \tilde{\kappa}=t$. Let $X \subseteq V_{1},|X|=\frac{1}{2}\left|V_{1}\right|$. Suppose all the pairs $\left(V_{i}^{(n)}, V_{j}^{(n)}\right), 1 \leq i<j \leq \nu-1$ are $\tilde{\varepsilon}$-regular. Put $m=\lfloor n / k\rfloor\left(\right.$ so $\left.\left|V_{i}\right| \sim m\right)$ and $F_{X}=G_{n}\left[X \cup V_{2} \cup \ldots \cup V_{\nu-1}\right]$ and $h=\left|X \cup V_{2} \cup \ldots \cup V_{\nu-1}\right|\left(\right.$ i.e. $\left.h \sim\left(\nu-\frac{3}{2}\right) m\right)$.

Now

$$
\mathbf{N}\left(L_{\nu} \subseteq F_{X}\right)=\mathbf{N}_{f}\left(L_{\nu} \subseteq F_{X}\right)+\mathbf{N}_{S}\left(L_{\nu} \subseteq F_{X}\right)
$$

where $N_{S}\left(L_{\nu} \subseteq F_{X}\right)$ denotes the number "copies" of $L_{\nu}$ covered by at most $(\nu-2)$ classes.

Applying (15) to $F_{X}=G_{n}\left[X \cup V_{2} \cup \ldots \cup V_{\nu-1}\right]$, we have

$$
\left|\mathrm{N}\left(L_{\nu} \subseteq F_{X}\right)-\gamma_{L}(p) h^{\nu}\right|<\varepsilon_{n} h^{\nu}
$$

\footnotetext{
6 Actually, we do not need here randomization: every partition $\left\{W_{\ell}\right\}$ obtained by joining small classes $V_{i(n)}^{n}$ will do with possibly $\varepsilon K^{2}$ exceptional pairs.

7 Usually we have to assume some lower bound on the densities $d\left(V_{i_{a}}, V_{i_{b}}\right)$, but here discarding the "poor" positions in the proof of the Main Lemma replaces this assumption.
} 
Using (15) also for the union of any $(\nu-2)$ classes we get with a $c_{1}$ depending only on $\nu$, on the densities $d_{i, j}$ and $\gamma_{L}(p)$, (but not on $X$ ) that

$$
\mathbf{N}_{S}\left(L_{\nu} \subseteq F_{X}\right)=c_{1} h^{\nu}+O\left(\varepsilon_{n}\right) h^{\nu}
$$

Hence, (by (16)) there is a $c_{2}$ independent of $X$ for which

$$
\left|\mathbf{N}_{f}\left(F_{X} \subseteq L_{\nu}\right)-c_{2} h^{\nu}\right|=O\left(\varepsilon_{n}\right) h^{\nu}
$$

On the other hand, applying Corollary 3.2 to $F_{X}$,

$$
\mathbf{N}_{f}\left(F_{X} \subseteq L_{\nu}\right)=A e(X) h^{\nu-2}+B h^{\nu}+O\left(\varepsilon_{n}\right) h^{\nu}
$$

where $A, B$ are constants (depending only on $\nu$ and on the densities $d_{i, j}, 1 \leq i<j \leq$ $\nu-1$ and on $\left.e\left(V_{i}\right), 2 \leq i \leq \nu-1\right)$ but do not depend on $X$. Hence, by (17) and (18), for any $X \subseteq V_{1},|X|=\frac{m}{2}$ we have with some constant $0 \leq \vartheta=\vartheta(n) \leq 1$ (independent of $X$ )

$$
e(X)=\vartheta|X|^{2}+O\left(\varepsilon_{n}\right)|X|^{2} .
$$

First we prove that if we restrict ourselves to "good" classes and $n \rightarrow \infty$, then

$$
\lim _{n \rightarrow \infty} \vartheta(n)=p \quad \text { uniformly. }
$$

Suppose indirectly, that there is a subsequence $s_{i} \rightarrow \infty$ such that

$$
\lim _{s_{i} \rightarrow \infty} \vartheta\left(s_{i}\right)=p^{*} \neq p
$$

Let us consider the graph sequence $\left(G_{s_{i}}\left(V_{1}^{\left(s_{i}\right)}\right)\right)$ where $V_{1}^{s_{i}}$ is a "good" class of the Szemerédi Partition belonging to the parameters $\varepsilon_{n}(=1 / t)$ and $\kappa_{n}=t$ for $n_{t}<s_{i} \leq n_{t+1}$. For $\left(G_{s_{i}}\left[V_{1}^{s_{i}}\right]\right), \mathbf{P}_{5}$ in Theorem A holds and consequently it is $p^{*}$-quasi-random. Therefore it contains $\gamma_{L}\left(p^{*}\right) s_{i}^{\nu} L_{\nu}$ 's. Since $\gamma_{L}(p)$ is strictly monotone increasing in $p$, this is a contradiction, proving Lemma 4.1: by Theorem A we get that the graph sequence $G_{n}\left[V_{1}^{n}\right]$ is $p$-quasi-random.

Proof of Lemma 4.2. Now we take the same setting as above, restrict ourselves to any "good" $\nu-1$-tuple, say to $(1,2, \ldots, \nu-1)$ and consider the graph sequence

$$
\left(G_{n}\left[V_{i}^{(n)} \cup V_{j}^{(n)}\right]\right), \quad i, j<\nu .
$$

We know already that $\left(G_{n}\left[V_{i}^{(n)}\right]\right)$ and $G_{n}\left[V_{j}^{(n)}\right]$ are $p$-quasi-random graph sequences. Let $F_{h}:=G_{n}\left[V_{i}^{(n)} \cup V_{j}^{(n)}\right]$. By $(*)$,

$$
\mathbf{N}\left(L_{\nu} \subseteq F_{h}\right)=\gamma_{L}(p) h^{\nu}+O\left(\varepsilon_{t}\right) h^{\nu}
$$


We assumed that the pair $\left(V_{i}^{(n)}, V_{j}^{(n)}\right)$ is $\varepsilon_{t}$-regular. Then by the strict monotonicity of $\mathrm{N}\left(L_{\nu} \subseteq F_{h}\right)$ in $d_{i, j}$ we get that for any fixed $\eta_{0}>0$

$$
\left|d_{i, j}-p\right|>\eta_{0}
$$

implies

$$
\left|\mathbf{N}\left(L_{\nu} \subseteq F_{h}\right)-\gamma_{L}(p) h^{\nu}\right|>\delta_{0} h^{\nu}
$$

with some fixed $\delta_{0}=\delta_{0}\left(\eta_{0}\right)>0$. This proves that

$$
\lim _{n \rightarrow \infty} d_{i, j}=p \quad \text { uniformly for any } \quad i(n), j(n) \in I_{n}
$$

if $I_{n}$ is an index-set of "good" classes.

Remark. The monotonicity, used at this very point makes the difference between this case and the case of the induced subgraphs: if we have a 2-class Generalized Random Graph $R_{n}$ with classes $V_{1}$ and $V_{2}$ and fix the edge-probabilities within the classes but vary the edge-probability $t$ between the two classes, then the expected number of copies of $L_{\nu} \subseteq R_{n}$ is a monotone increasing function of $t$ in case of NNI copies but it is not monotone in the Induced Case.

\section{Open problems}

1. Is it true that when we wish to prove the quasi-randomness assuming that each $F_{h}$ of each $G_{n}$ contains $\left.\gamma_{(} p\right) h^{3}$ triangles, in that place it is enough to assume the condition for each $n$ for 2 or 3 appropriate values of $h$ ?

2. It would be interesting to know if there is a direct proof of our theorems, say of Theorem 2.4 not using the notion of $\varepsilon$-regularity, or Regularity Lemma, in any form.

\section{References}

[1] B. BollobÁs: Random Graphs, Academic Press, 1985.

[2] F. R. K. Chung, R. L. Graham and R. M. Wilson: Quasi-random graphs, Combinatorica, 9 (4), (1989), 345-362.

[3] F. R. K. Chung, R. and R. L. Graham: Quasi-random hypergraphs, Random Structures and Algorithms, 1 (1990), 105-124.

[4] F. R. K. Chung: Regularity lemmas for hypergraphs and quasi-randomness, Random Structures and Algorithms, Vol. 2 (2) (1991), 241-252.

[5] F. R. K. ChUNG and R. L. Graham: Quasi-random set systems, Journal of the American Math. Society, 4 (1) January, (1991), 151-196. 
[6] F. R. K. Chung and R. L. Graham: Maximum cuts and quasi-random graphs, in Random Graphs, (Poznan Conf, 1989) Wiley-Intersci, Publ. vol 2, 23-33.

[7] F. R. K. Chung and R. L. Graham: On hypergraphs having evenly distributed subhypergraphs, (in the Proc. Conf. Marseille-Luminy, 1990) Discrete Mathematics, 111 (1-3) (1993), 125-129.

[8] M. DYER and A. FRIEZE: Computing the volume of convex bodies: a case where randomness provably helps, in Probabilistic Combinatorics and Its Applications (ed. Béla Bollobás), Proceedings of Symposia in Applied Mathematics, Vol. 44 (1992), 123-170.

[9] P. FRANKL, V. RöDL AND R. M. WILSON: The number of submatrices of given type in an Hadamard matrix and related results, Journal of Combinatorial Theory, (B) 44 (3) (1988), 317-328.

[10] A. N. Kolmogorov: Three approaches to the quantitative definition of information, Problems Inform. Transmition, 1 (1965), 1-7.

[11] J. Komlós, G. N. SÁrKözy and E. Szemerédi: Blow-up Lemma, Combinatorica, 17 (1) (1997), 109-123.

[12] J. Komlós and M. Simonovits: Szemerédi Regularity lemma and its applications in Extremal Graph Theory, in: Paul Erdös is 80, II. Bolyai J. Math. Soc. 2, (1996), 295-352.

[13] M. Simonovits and V. T. Sós: Szemerédi's Partition and quasi-randomness, Random Structures and Algorithms, 2 (1991), 1-10.

[14] M. Simonovits and V. T. Sós: Hereditarily extended properties, quasi-random graphs and induced subgraphs, manuscript.

[15] E. SzemerÉDi: On regular partitions of graphs, Problemes Combinatoires et Théorie des Graphes (ed. J. Bermond et al.), CNRS Paris, 1978, 399-401.

[16] E. SzEMERÉDI: On graphs containing no complete subgraphs with 4 vertices (in Hungarian) Mat. Lapok 23 (1972), 111-116.

[17] A. Thomason: Random graphs, strongly regular graphs and pseudo-random graphs, in: Surveys in Combinatorics, 1987 (Whitehead, ed.) LMS Lecture Notes Series 123, Cambridge Univ. Press, Cambridge, 1987, 173-196.

[18] A. Thomason: Pseudo-random graphs, in: Proceedings of Random graphs, Poznan, 1985, (M. Karonski, ed.); Annals of Discrete Math., 33 (1987), 307-331.

[19] A. Thomason: A disproof of a theorem of Erdös in Ramsey theory, J. London Math. Soc, 39 (2) (1989), 246-255.

Miklós Simonovits

Mathematical Institute of the

Hungarian Academy of Sciences,

Budapest, Hungary

miki@math-inst.hu
Vera T. Sós

Mathematical Institute of the

Hungarian Academy of Sciences,

Budapest, Hungary

sos@math-inst.hu 